\title{
Medicamentos viejos para pacientes nuevos: el impacto de la crisis en los servicios sociales de la ciudad de Zaragoza desde la perspectiva de los trabajadores sociales ${ }^{1}$
}

\author{
Elisa Esteban Carbonell ${ }^{2}$ \\ Universidad de Zaragoza \\ <estecar@unizar.es>
}

\section{Sandra Romero Martín \\ Universidad de Zaragoza}

\section{$M^{\mathbf{a}}$ del Mar Del Rincón Ruiz}

Universidad de Zaragoza

\begin{abstract}
Artikulu honetan aztergai hartzen da Zaragozako gizarte-zerbitzuetako lehen mailako arretan (edo gizarte-zerbitzuetan) krisialdiak eskuartze sozialetan izandako eraginen inguruko gizarte-langileen diskurtsoa. Gertatu diren aldaketa horiek aztertzen dira ondorengo hiru alderdi hauetan: artaturiko erabiltzaileak (kopurua, ezaugarriak, aurkeztutako eskariak, beste sistema batzuetan eman diren murrizketek gizarte-zerbitzuetan izandako eragina); egungo eskariei aurre egiteko zerbitzuen erantzuteko gaitasuna (arretari eskainitako astia, baliabideen nahikotasuna, itxaronaldiak, prestaziosistemaren ebaluazioa, premia berriei eskainitako erantzuna, komunitatearen ekimenak, gizarteekintzako elkarte pribatuen rola, beste premiekiko atentziorik eza); eta balorazio globala eta proposamenak. Azterlanak oinarri hartzen ditu 2013 eta 2016ean sakonean eginiko hamabi elkarrizketa, eta horrek aukera eskaintzen du bi aldi horietako egoeren arteko konparazioa burutzeko.
\end{abstract}

\section{GAKO-HITZAK:}

Lehen mailako gizarte-zerbitzuak, Zaragoza, krisialdia, eskuartze soziala, erabiltzaileak.

\begin{abstract}
Este artículo analiza el discurso de los trabajadores sociales sobre los efectos de la crisis en la intervención social en los servicios sociales públicos de atención primaria (o servicios sociales generales) de la ciudad de Zaragoza. Se examinan los cambios producidos en tres aspectos: los usuarios atendidos (volumen, características, demandas planteadas, impacto en los servicios sociales de los recortes de otros sistemas); la capacidad de respuesta de los servicios a las demandas actuales (tiempo dedicado a la atención, suficiencia de recursos, tiempos de espera, evaluación del sistema de prestaciones, respuesta a las nuevas necesidades, iniciativas de la comunidad, papel de las entidades privadas de acción social, coordinación de los servicios sociales con las entidades privadas, desatención de otras necesidades); y valoración global y propuestas. El estudio se basa en doce entrevistas en profundidad realizadas en 2013 y 2016, lo que permite también comparar la situación en esos dos momentos.
\end{abstract}

\section{Palabras Clave:}

Servicios sociales de atención primaria, Zaragoza, crisis, intervención social, usuarios.

\footnotetext{
${ }^{1}$ Este artículo parte de una comunicación presentada en el XII Congreso Español de Sociología (Gijón, 2016).

${ }^{2}$ Becaria predoctoral del Gobierno de Aragón. Parte de este trabajo se ha realizado, por tanto, con el apoyo del Gobierno de Aragón.
} 


\section{Introducción}

Los servicios sociales han sufrido importantes cambios en los últimos años a partir de los efectos de la crisis. En este sentido, junto con el aumento del número de usuarios atendidos y la diversificación de sus características, el sistema de servicios sociales debe hacer frente a mayor número de demandas con menor número de recursos. Además, la falta de cobertura del resto de sistemas deja al descubierto necesidades básicas que deben ser cubiertas por los servicios sociales. Esta realidad está sobrecargando al sistema, que se ve incapaz de hacer frente a esta situación sobrevenida. Por otra parte, estamos asistiendo a un aumento de las prestaciones económicas frente a las prestaciones de servicio, y este cambio de prioridades ha modificado el quehacer profesional. Por todo ello, es imprescindible conocer la percepción de los profesionales que se encuentran día a día con esta realidad, para comprender la situación que atraviesan los servicios sociales.

El presente artículo muestra los resultados de una investigación principalmente cualitativa llevada a cabo en los centros municipales de servicios sociales del Ayuntamiento de Zaragoza en dos fases (2013 y 2016), con el objetivo de conocer el impacto de la crisis sobre los servicios sociales respecto a los usuarios y al tipo de intervención.

\section{Metodología}

La investigación parte de la observación de que la atención a los usuarios en los servicios sociales públicos de atención primaria (o servicios sociales generales) ${ }^{3}$ de la ciudad de Zaragoza se ha visto afectada a raíz de la crisis. La primera fase, realizada en 2013 en el marco del trabajo final de grado en trabajo social, tenía una doble finalidad. Por un lado, conocer las consecuencias de la crisis en las áreas de empleo, vivienda, salud, educación y dependencia, así como la repercusión que dichos efectos estaban teniendo sobre el sistema de servicios sociales. Y por otro lado, analizar la opinión y la percepción de los profesionales en torno a la intervención con los usuarios.

Partiendo de los resultados obtenidos, se retomó la investigación, llevando a cabo una segunda fase tres años más tarde para contrastar la situación de los servicios sociales en ambos momentos. La hipótesis

${ }_{3}^{3}$ Tal y como recoge el artículo 13 de la Ley 5/2009, de 30 de junio, de Servicios Sociales de Aragón:

1. Los servicios sociales generales son servicios públicos de carácter polivalente y comunitario, cuya organización y personal están al servicio de toda la población.

2. Los servicios sociales generales constituyen el primer nivel del sistema público de servicios sociales y proporcionan una atención que tiene por objeto favorecer el acceso a los recursos de los sistemas de bienestar social, generando alternativas a las carencias o limitaciones en la convivencia, favoreciendo la inclusión social y promoviendo la cooperación y solidaridad social en un determinado territorio. de partida era que la demanda había continuado incrementándose, llegando incluso a la cronificación de ciertas situaciones, al mismo tiempo que el sistema de servicios sociales continuaba saturado y con instrumentos 'cortoplacistas', sin adaptarse a las nuevas situaciones. El presente artículo muestra los resultados de ese análisis comparativo.

Dada la complejidad del fenómeno a estudiar, se decidió emplear la técnica de la entrevista para la parte cualitativa de la investigación. Con el fin de concretar el objeto de estudio, se decidió identificar tres dimensiones que sirvieron para estructurar el guión de las entrevistas:

- Usuarios de los servicios sociales generales: variación en el volumen de los usuarios atendidos, características, demandas planteadas e impacto en los servicios sociales de los recortes de otros sistemas.

- Capacidad de respuesta de los servicios sociales a las demandas actuales: tiempo dedicado a la atención, suficiencia de recursos, tiempos de espera, evaluación del sistema de prestaciones, respuesta a las nuevas necesidades, iniciativas de la comunidad, papel de las entidades privadas de acción social, coordinación de los servicios sociales con las entidades privadas y desatención de otras necesidades.

- Valoración global y propuestas: detección de los principales problemas de los servicios sociales y propuestas de mejora.

En total, se llevaron a cabo doce entrevistas, nueve de ellas en la primera fase (2013) y tres en la segunda (2016). De las iniciales, dos fueron abiertas y dirigidas a profesionales de Cáritas Diocesana de Zaragoza, y las siete restantes, semiestructuradas y a trabajadores sociales de los servicios sociales del Ayuntamiento de Zaragoza, al igual que las tres entrevistas posteriores. Las entrevistas, que se grabaron y transcribieron en su totalidad, estaban orientadas a conocer los aspectos más valorativos sobre la situación actual de los servicios sociales. Su contenido se analizó de forma extendida basándose en las dimensiones planteadas.

\section{Marco teórico y contextual}

Muchas publicaciones muestran que la crisis iniciada en 2008 está generando cambios en nuestra sociedad (Fundación 1ํ de Mayo, 2012; Fundación Foessa, 2012, 2013; y Observatorio Estatal de la Dependencia, 2012; entre otras), y tanto sus efectos como las políticas desarrolladas han producido consecuencias notables en varios pilares de nuestro sistema de bienestar, entre los que se encuentran los servicios sociales. Algunas de las consecuencias más notables o más analizadas son el empobrecimiento de la población en general y la reaparición del fenómeno que se creía olvidado, o superado, como es el de los trabajadores pobres (Fundación 1ํ de Mayo, 2012). 
Como señala Fantova (2016: 115), el análisis de las causas, efectos, cuantías y dimensiones de esta afectación ha sido realizado en otros trabajos (Asociación Estatal de Directoras y Gerentes de Servicios Sociales, 2016; Ayala y Ruiz-Huerta, 2015: 258-268; Barbieri y Gallego, 2015; Barriga, 2014; Deusdad, 2016; Laparra y Pérez Eransus, 2012; Lima, 2013; López Casasnovas, 2015; Martínez Virto y Pérez Eransus, 2015; Montserrat, 2015; Rodríguez Cabrero, 2012; Rodríguez Rodríguez, 2015; y Sanzo, 2013).

Por un lado, se puede hablar de consecuencias derivadas de otros sistemas. Los efectos de la crisis en un modelo desarticulado pueden ser devastadores para los servicios sociales, ya que al incremento de la demanda, derivado del envejecimiento de la población y de la consecuente implantación del Sistema de Atención a la Dependencia, hay que añadir la explosión de necesidades sociales insatisfechas por el efecto del desempleo, de las reducciones salariales y de la crisis de la vivienda" (Alguacil Gómez, 2012: 72). Así, se ha podido constatar cómo la crisis, con su 'efecto dominó', ha generado nuevas necesidades en los servicios sociales 0 , dicho de otra manera, necesidades no resueltas por otros sistemas han llegado al de los servicios sociales.

La Figura 1 recoge de manera simplificada las principales consecuencias sobre el sistema de servicios sociales detectadas en la investigación inicial.
El aumento en el número de personas sin cobertura por parte del sistema de empleo, así como la disminución de las posibilidades económicas, ya sea por la pérdida de empleo o por la precariedad laboral, repercute directamente en el sistema de servicios sociales, en forma de incremento de demandas y casos atendidos: "los profesionales de los servicios sociales consultados [en 2012] cifran este incremento de usuarios entre un $50 \%$ y un $300 \%$ desde que comenzó la crisis" (Marea Naranja Aragón, 2012: 21). Parte de los usuarios solicitan el ingreso aragonés de inserción (IAI) como última vía de obtención de ingresos, una vez agotado otro tipo de prestaciones (contributivas, asistenciales o renta activa de inserción): "en tres años y medio, se ha multiplicado por siete el número de perceptores del ingreso aragonés de inserción” (ibídem: 22).

Para hacer frente a los crecientes problemas de vivienda por la pérdida de poder adquisitivo de los hogares, éstos pueden solicitar ayudas de urgencia a los servicios sociales. El número de estas ayudas ha aumentado en los últimos años. Las ayudas de urgencia también se utilizan para apoyar a las familias en el pago del comedor infantil, lo cual compensa la reducción de las becas. Por ello, los servicios sociales comunitarios del Ayuntamiento de Zaragoza han estado gestionando ayudas de urgencia dentro del Plan Integral Casco Histórico (PICH) y del Plan Integral Barrio Oliver (PIBO) 4 . Los servicios sociales también tramitan ayudas de urgencia para cubrir necesidades sanitarias.

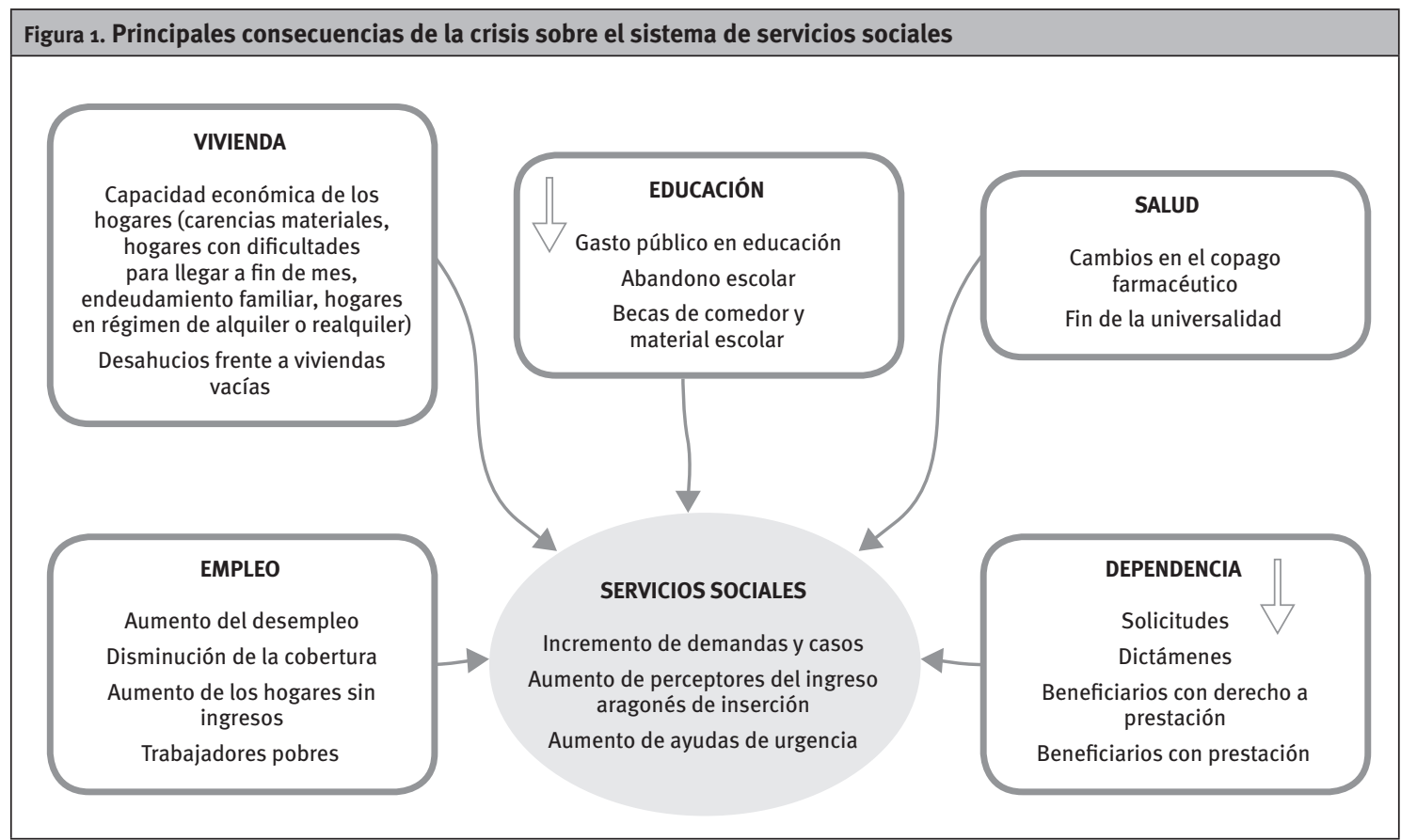

Fuente: Elaboración propia. 
Hasta 2012, los servicios sociales municipales gestionaban este tipo de ayudas para complementar al sistema de Seguridad Social en aspectos que éste no cubría (prótesis dentales, gafas) ${ }^{5}$.

Todo ello muestra cómo la deconstrucción del Estado del bienestar asociada a la crisis configura unas nuevas coordenadas de ubicación del trabajo social. Ahora bien, este proceso de ajuste, lejos de ser automático, exige alguna forma de reflexión y autoconceptualización por parte del propio trabajo social, o mejor aún, por parte del trabajador/a social en situación (Abad Miguélez y Martín Aranaga, 2015: 180).

Por otro lado, y sin olvidar la idea inicial, encontramos unos servicios sociales tardíamente desarrollados e incapaces de poner en marcha herramientas eficaces y de hacer frente a esta nueva situación. En general, el modelo de atención de los servicios sociales de base se ha burocratizado y esclerotizado (Duque Carro, 2012: 24). La burocratización es, precisamente, uno de sus hándicaps más importantes. La práctica mayoritaria de tramitación de prestaciones económicas ha conformado una práctica profesional de tipo administrativo, caracterizada por el trabajo de despacho y las tareas de comprobación de requisitos, situándose así muy lejos de la dinámica relacional individual y familiar, y aún más, de las dinámicas comunitarias (ibídem: 25).

La nueva realidad trae consigo unas nuevas necesidades basadas fundamentalmente en la atención a necesidades básicas, como señala, entre otros, Aguilar Hendrikson ${ }^{6}$. Sin embargo, no debemos olvidar que la intervención de los trabajadores sociales debería ir mucho más allá. Es necesario, en este sentido, una reflexión conjunta sobre la situación actual que viven los servicios sociales, que detecte aquellos aspectos susceptibles de ser redireccionados. El trabajo aquí presentado pretende contribuir a ese análisis en un nivel micro (los servicios sociales generales de la ciudad de Zaragoza), con el objetivo de retratar aquellas modificaciones más significativas de su funcionamiento a partir de la crisis, y dejar la puerta abierta para una investigación posterior que ahonde y profundice en soluciones.
${ }_{5}^{5}$ Sin embargo, a partir de enero de 2013 una instrucción interna suspendió las ayudas bucodentales que no constituían un problema sanitario (consideradas no básicas) y las ópticas (concedidas excepcionalmente).

6 "Desde 2008, la crisis inmobiliaria y financiera y su cadena de efectos devastadores sobre el empleo y la hacienda pública [...] han sepultado a los servicios sociales bajo una avalancha de demandas de ayuda para la subsistencia” (Aguilar Hendrikson, 2013: 147).

\section{Resultados de la investigación}

\subsection{Usuarios}

En este apartado, se analiza la información recabada sobre los usuarios de servicios sociales: variaciones en el volumen de usuarios atendidos, características, demandas presentadas y prioridad de éstas, así como el posible impacto en los servicios sociales como consecuencia de los recortes de otros sistemas de bienestar (o ramas de actividad del sistema de bienestar).

En cuanto a la variación en el volumen de los usuarios atendidos, todos los entrevistados, tanto de la primera fase de la investigación como de la más reciente, señalan que el aumento de usuarios en el último tiempo ha sido considerable. E2, E4 y E5 coincidían en que "ha aumentado de forma exponencial”, y algunos se arriesgaban a precisar el tamaño de ese acrecentamiento. Si bien E2 afirmaba que "los beneficiarios de determinadas prestaciones sociales se ha[n] llegado a multiplicar por 7 [...]”, E4 aportaba porcentajes más concretos y aseguraba que, en su centro, “en el año 2012, [...] el número de expedientes nuevos abiertos se incrementó en un 30\%". En esta línea, es interesante el dato aproximado que aporta E12, al señalar que, en el centro donde desarrolla su actividad, en diez años se han abierto cerca de 4.000 nuevos expedientes, si bien no todos se encuentran activos.

Todos los entrevistados ponen en evidencia el aumento de usuarios, y algunos se refieren también a otros indicadores. E1 mencionaba el aumento de "la complejidad de la demanda", y $E_{5}$ se adelantaba a la siguiente pregunta al afirmar que "ha aumentado no sólo el número, sino los perfiles”. En cualquier caso, lo relevante no es tanto la precisión del aumento, sino la percepción que tienen los trabajadores sociales sobre él, pues, desde su punto de vista, se traduce automáticamente en mayor carga de trabajo.

En lo que respecta a las características de los usuarios, todos los entrevistados distinguían y siguen distinguiendo "los colectivos tradicionales", la "población clásica", los "colectivos de antes" o los "colectivos anteriores" de los nuevos colectivos. No importa tanto el calificativo que le otorgan, sino la distinción entre los usuarios habituales de servicios sociales y los nuevos usuarios del sistema.

Asimismo afirmaban la existencia de este emergente grupo que, o bien era nuevo completamente en los servicios sociales o, como señalaba E2, acudía “de forma muy esporádica”. En la misma línea opinaba E9 al decir que eran habitualmente "personas o que no habían tenido relación con servicios sociales, o que, si la habían tenido, ésta había sido muy puntual”, y que además, tal y como afirmaba E7, "llegan a estar en un punto de exclusión social, de riesgo de exclusión social grave”. 
Estos dos grupos de usuarios atendidos por los servicios sociales se han mantenido en el tiempo, y así lo ponen de manifiesto los recientes entrevistados. En relación con los colectivos tradicionales de servicios sociales, es interesante señalar que ahora se encuentran en "una situación de mayor precariedad que la anterior, porque ahora hay mayor competencia, incluso para el acceso a servicios sociales" (E11).

Respecto a estos nuevos usuarios, aun habiendo diferentes opiniones al respecto, se trata sólo de pequeños matices, puesto que todos señalan el empleo como factor clave en la aparición de este colectivo. En este sentido, el desempleo, y más concretamente, el desempleo de larga duración, continúa siendo en 2016 el motivo de llegada de los nuevos usuarios, o mejor dicho, la razón por la que estos colectivos emergentes siguen siendo atendidos en los centros.

Lo interesante es que los usuarios de hoy en día presentan características distintas a los 'clásicos usuarios':

Hasta hace cinco años más o menos, que sería el 2008, [...] había un tipo de usuario o un perfil de usuario en el que se daban dos características: [...] un problema económico [y] un problema de carácter personal/social que le impedía llevar una vida autónoma, organizada y propia (E5).

Como varios de los informantes señalaban, entre ellos, E9, “[su] único problema es el económico”, porque en otras áreas no presentan debilidades; añadía que "tienen todos los hábitos, saben buscar empleo, se organizan bien en casa..., pero se han quedado sin dinero". Aunque es la ausencia de ingresos el denominador común entre los ‘clásicos' y los 'nuevos' usuarios, estos últimos “no necesitan un plan de intervención, [...] no hace falta un trabajador social”.

Esta misma percepción la tienen los nuevos trabajadores sociales entrevistados: la carencia de ingresos, como consecuencia evidente de la falta de un empleo y de protección social, supone la puerta de entrada al sistema de servicios sociales. Sin embargo, también observan otro tipo de características, como un "deterioro personal y emocional muy importante” (E11).

Otra diferencia palpable entre ambos momentos de la investigación y, evidentemente, entre los usuarios de uno y otro, se refiere a la información. Los recientes entrevistados señalan que parte de los usuarios que llegan hoy en día a sus centros están más informados, ya no tanto sobre el propio sistema de servicios sociales o sobre el centro7, sino más

7 "Mucha gente viene derivada del Inaem y vienen con la idea de que es otro ente de la Administración [...], con la idea de 'vengo a presentar aquí mi subsidio...'. Entonces le explicas que es otro mundo, que la valoración es de otra manera, y eso a veces causa per- bien sobre las prestaciones y recursos que desde ahí se ofrecen, bien porque han buscado ellos mismos información al respecto ${ }^{8}$, o bien por el boca a boca 9 . Además, también puede tratarse de "un perfil de ciudadanos conocedores de derechos que tienen" (E12), situación que, como estos profesionales remarcan, modifica la relación entre el trabajador social y el usuario, que pasa de ser jerárquica a presentar un equilibrio entre ambas posiciones.

También se constata que los servicios sociales estaban "acostumbrados a un determinado perfil de personas que tenían ciertos déficits y [...] lo que hacía[n] era intentar que desarrollaran habilidades para que esos déficits desaparecieran..." (Eg).

En cuanto a las demandas de los usuarios, E1 y E2 coincidían en que se estaba produciendo un cambio en la priorización de las necesidades, de modo se intervenía con el fin de cubrir necesidades de subsistencia. Así pues, se estaba relegando el trabajo social realizado hasta el momento, que "ha quedado reducid[o] a su mínima expresión” (E2).

Varios de los entrevistados daban un listado de las cuatro principales necesidades y, observando los datos, por lo general las ayudas económicas dirigidas a cubrir las necesidades básicas ${ }^{10}$ se encontraban en primer lugar. Entre ellas, los problemas de vivienda cobraban una importancia cada vez mayor: "el mantenimiento de la vivienda está siendo el principal problema de las personas en situación de desempleo o aquejadas por la crisis" $\left(E_{5}\right)$.

Lo que resulta relevante señalar es que casi todos ellos observaban un cambio en el tipo de intervención que antes brindaban los servicios sociales. De este modo, no se actuaba de manera integral, sino tratando de remediar tan sólo algunos de los déficits que presentaban los usuarios, al intervenir únicamente sobre la ausencia de ingresos, que, tal como se ha señalado, era y es el denominador común entre los usuarios nuevos y los tradicionales.

Una opinión similar mantienen los nuevos entrevistados y, concretamente, señalan que el tipo de demandas ahora son económicas:

En el centro de servicios sociales, la gente acude por la oferta que ellos entienden que hay... [...] un pequeño grupo que es por problemática de menores...; está el grueso de gente mayor, que

plejidad..., porque la gente viene con la idea de 'ahora me toca este subsidio"” (E10).

8 "Otra gente, mucha gente, cada vez más gente está buscando por internet información, entonces vienen... Pero internet no te da la información tal cual, no te prepara para lo que es ir al centro de servicios sociales" (E10)

9 “Otra gente viene... 'me he enterado', 'mi vecina cobra ayuda, me ha dicho ‘pásate a ver que hay’, ¿no?...” (E10).

${ }_{10}$ Para mayor información, véase 〈http://www.aragon.es/DepartamentosOrganismosPublicos/OOAA/IASS/Inicio/AreasActividad/ ServiciosSociales/ci.ssgrales_seccion_09_ayudas_urgencia.detallelnaem>. 
es por dependencia, prestaciones; y el resto de gente siempre es por una demanda económica. Siempre. Que antes era más otra población, y ahora es también población normalizada... (E10).

Entre las ayudas de urgente necesidad, se ha constatado un aumento en aquellas destinadas a cubrir gastos de hipoteca: "en general, las personas que tradicionalmente se atendían en servicios sociales no optaban a hipotecas, y en estos momentos sí que nos encontramos con más ayudas de esas características" (E11).

En relación con las consecuencias en los servicios sociales de los recortes en otros sistemas de bienestar, todos señalaban cómo estaban afectando de manera preocupante en la intervención de los servicios sociales, ya que cuando no existe protección por parte de alguno de los sistemas, el usuario acude inmediatamente a servicios sociales, por ser "la última red de protección” (E1). De este modo, se observaba una saturación de los servicios, incapaces de resolver las demandas recibidas, unida a unos recortes que aumentaban progresivamente también en esta área: "la paradoja es que en los servicios sociales también está habiendo recortes directa [más requisitos para acceder a determinados programas] o indirectamente [más tiempo en resolver solicitudes de determinados programas]” (E3).

En cualquier caso, los servicios sociales estaban cubriendo déficits propios de otras áreas, principalmente "la retirada de la asistencia sanitaria a extranjeros sin permiso de residencia [y] el recorte en becas de comedor escolar, material, etc." (E2). Sin embargo, los entrevistados en 2016 ponen el foco en los cambios en el sistema de adjudicación de las becas educativas, con recortes en la cobertura que recaen en los servicios sociales ${ }^{11}$. Si bien dicen que posiblemente los recortes se han frenado, los requisitos de acceso a las becas se han endurecido, de modo que dejan grupos de población que, pese a contar con pocos recursos, no pueden acceder a una beca escolar.

Por otra parte, en el área de vivienda se han conseguido claros avances, y así lo manifiestan los profesionales. Inicialmente, fueron plataformas o movimientos sociales, como Stop Desahucios, quienes demandaron y consiguieron algunos cambios legales (código de buenas prácticas de los bancos, por ejemplo), y posteriormente se han visto modificaciones sustanciales por parte de las Administraciones, como el caso del Ayuntamiento de Zaragoza, que ha puesto en marcha un programa de mediación en deuda hipotecaria a partir de Zaragoza Vivienda.

Sin embargo, a pesar de estos tímidos avances, se está obligando a que los servicios sociales

\footnotetext{
${ }^{11}$ Para más información, véase D. L. G. (2016).
}

intenten solucionar un problema que es del propio sistema..., es decir, cuando todo se tambalea, ahora, tienen que salir al rescate los servicios sociales [...]. Que lo que no cubre ningún sistema de protección del Estado del bienestar, lo cubran los servicios sociales, [que han sido] pensados para una población marginal (E8).

En este sentido, no podemos olvidar que "la respuesta a la crisis no está en los servicios sociales" (E4).

\subsection{Capacidad de respuesta de los servicios sociales a las demandas actuales}

Este apartado aborda la atención dispensada (tiempos de atención y espera, recursos) y el papel desempeñado por las iniciativas comunitarias y entidades privadas, así como la coordinación entre éstas y los servicios sociales generales. También busca conocer la valoración sobre la atención prestada, así como sobre la actuación de la Administración, en el ámbito de los servicios sociales.

En cuanto al tiempo dedicado a la atención de cada usuario, los entrevistados coincidían en que era "absolutamente insuficiente" (E2), "lo que hace que el estrés esté asegurado en nuestro quehacer cotidiano" (E3). Esta situación se debía al aumento de la demanda ${ }^{12}$, pero también a la carencia de recursos humanos suficientes para hacerle frente ${ }^{13}$ Todo ello se une al plan de choque puesto en marcha por el Ayuntamiento de Zaragoza para tratar de hacer frente al colapso de los servicios sociales:
Se nos inventaron [...] un 'plan de choque' que consistía en que nuestras citas tienen que ser de veinte minutos... Entenderéis que intervención social, realmente, en veinte minutos puedes hacer muy poca, porque simplemente contextualizas un poco [...] la situación de esa persona, qué es lo que necesita administrativamente [...]. Puedes intentar hacer una atención lo más correcta posible, pero no es intervención social, es otra cosa (E11).

Hay profesionales que efectivamente cumplen la directriz marcada, pero

trabajar así significa: ‘qqué quiere usted?’, ‘una ayuda de luz', 'deme los papeles...', la tramito y ya está. La gente, entre la que yo me considero, que tenemos muy claro lo que es la trabajadora social, es imposible atender a nadie en veinte minutos... ¿Qué es lo que pasa? Que para hacer esa atención, mínimamente digna, no voy a decir

\footnotetext{
${ }_{12}$ "El número de familias te ha aumentado, y donde antes tenías que trabajar con veinte, ahora tienes que trabajar con cien” (E6).

${ }_{13}$ "En este momento no se cubren bajas, no se cubren excedencias, la demanda se ha multiplicado y estamos los mismos, con lo cual, pues la atención es cada vez peor, es así” (E7).
} 
que sea perfecta..., es... a costa de un desgaste personal muy grande... (E10).

A pesar de que todos coincidían en que debía primar la calidad del servicio, sus respuestas variaban. De este modo, E4 explicaba que intentaba llevar a cabo "una intervención cualitativa que, aunque sea en pocos minutos, no olvide los aspectos emocionales y relacionales de las personas". E1 explicaba que el aumento de la demanda la había obligado a priorizar la atención del mayor número posible de casos, “en detrimento, lógicamente, de la calidad de la intervención”. No obstante, explicaba su descontento con la situación, manifestando que esta decisión la hacía muchas veces "ir a casa cabizbaja".

Aunque la atención de veinte minutos sigue estipulada, se están produciendo pequeños cambios organizacionales:

"Desde el cambio de Gobierno ${ }^{14}[$ [...], se está trabajando [...] en [...] la estructura de servicios sociales comunitarios, [...] de una forma participativa, para intentar que se trabaje por programas, [...] Pero por el momento, no ha habido cambios efectivos" (E11).

Además de estos problemas organizativos, los profesionales también evidencian cómo, en ocasiones, tienen que poner en valor su propia profesión en aspectos relativos a la atención y al tiempo que le dedican:

Cuando hablas estas cosas, hay quien... entiende y hay quien no. Por ejemplo..., nuestro trabajo es muy ingrato, porque..., en mi propio centro, los administrativos no entienden para qué queremos tiempo después. 0 sea..., la idea es que tú estás atendiendo y estás sacando el trabajo... que después, como... hace poco una técnico auxiliar dijo..., 'pero luego, después, ¿para qué quieren el tiempo las trabajadoras sociales...? Entonces, sientes impotencia, pero es que nadie entiende cómo funciona esto..., que la gente se debe de pensar que la gente entra, le das a un botón... y ya están las cosas hechas... Y ni siquiera las ayudas son fáciles de tramitar (E10).

Como se ha señalado, los profesionales eran y son totalmente conscientes de que la calidad en la atención a los usuarios de servicios sociales "tendría que ser un parámetro a tener muy en cuenta”, como señalaba E2, y que "la mayor parte de los casos atendidos requerirían de más tiempo tanto de atención directa como de reflexión posterior sobre el plan de intervención a seguir” (E3).

En relación con la suficiencia de los recursos frente a las demandas, cuatro de los primeros entrevistados manifestaban que no se estaban

${ }_{14}$ Como resultado de las elecciones municipales de mayo de 2015, el Ayuntamiento de Zaragoza pasó de manos del PSOE a las de Zaragoza en Común. destinando suficientes recursos para la atención adecuada a las demandas realizadas. Como ejemplo, E3 señalaba que "sí se echan de menos recursos a nivel formativo-laboral, y se nota que han ido a menos, especialmente con la crisis (recortes de servicio de orientación laboral, oferta de proyectos de cualificación profesional, etc., etc.)”. Por su parte, E6 opinaba que, en áreas como vivienda, "sí que están poniendo en marcha, ahora la DGA [Diputación General de Aragón] la red de bolsas de vivienda de alquiler social y tal. También están las ayudas de inquilinos", pero consideraba que otros recursos eran insuficientes, como el ingreso aragonés de inserción, que "hoy por hoy, está mal”. Esta última situación, sin embargo, se ha visto mejorada sustancialmente a partir del cambio de Gobierno autonómico ${ }^{15}$, como ponían de manifiesto los recientes entrevistados.

No obstante, varios de los entrevistados iniciales, así como los tres profesionales entrevistados posteriormente, coincidían en que, más allá de los recursos, existía un déficit en la organización del propio sistema, siendo "muy inadecuada la organización actual de los servicios sociales” (E2). En este sentido, la solución "no sería tanto crear muchos recursos más, sino que funcionen bien los que existen”, como apuntaba E3 o en el momento actual E11: "porque a lo mejor lo que necesitaríamos es que cada uno de esos recursos funcionase, 0 de esos sistemas funcionase como tendría que funcionar". Por otra parte, la falta de coordinación entre Administraciones repercutía en que el Ayuntamiento de Zaragoza asumiera indirectamente los recortes del Gobierno de Aragón: “el Gobierno de Aragón recorta becas, ¿la persona que hace? Como no puede, compra los libros de los críos y luego no tiene o para comer o para pagar la luz. ¿Dónde va? Al Ayuntamiento. El Ayuntamiento está pagando indirectamente el recorte de la DGA" (E5).

En cuanto a la pregunta sobre qué haría falta para una buena atención, $E_{5}$ defendía que, en primer lugar, sería urgente un aumento de los recursos: "es que [se] está destinando calderilla, hay que destinar más. Es así. En tiempos de crisis hay que dedicar más. Cuando no hay crisis, es cuando no hace falta, porque la gente se busca la vida". Por otro lado, tal y como señalaba E1, la prioridad era agilizar los servicios: “las prestaciones y servicios deben ser lo más ágiles posibles y adecuarse tanto las cuantías como los tiempos a las necesidades existentes, a la realidad actual”. Además, para el E5, era importante adaptar la respuesta a las demandas actuales de los ciudadanos: "en tiempo de crisis, las administraciones públicas tienen que priorizar al ciudadano y tienen que conocer cuál es la realidad y hacer frente a las necesidades que tenemos los ciudadanos".

${ }_{15}$ Como resultado de las elecciones autonómicas de mayo de 2015 el Gobierno de Aragón pasó de manos del PP a manos del PSOE (en coalición con CHA). 
Para conseguir estos objetivos, los trabajadores sociales consultados planteaban diversas respuestas. En primer lugar, según los profesionales, era necesaria una reflexión previa: "sería necesaria una visión estratégica. ¿Qué es lo que queremos que hagan los servicios sociales?: ¿un repartidero de ayudas?, ¿o tendrían que situarse en ese territorio que sobrepasa lo individual para buscar lo colectivo, el apoyo social?" (E4). Por otra parte, E3 manifestaba la necesidad de "modificar la 'atención a demanda' hacia la 'atención a nivel integral' [...], planteando los recursos como herramienta, como medios, no como fin, lo que daría más calidez y calidad a dicha atención/intervención".

En cuanto a la búsqueda de lo colectivo y del apoyo social, podría llevarse a cabo mediante "planes de acción coordinados entre instituciones del ámbito social, el empleo, la sanidad, educación, vivienda" (E1). Además, E1 manifestaba otros dos elementos claves para la mejora de la atención: la mejora de la coordinación entre profesionales, de manera que éstos no siguieran "trabajando aislados", así como el aumento de la iniciativa - "no podemos seguir siendo reactivos"-.

Por su parte, los recientes entrevistados también aportan alguna propuesta de mejora interesante al respecto, como por ejemplo:

Haría falta una comprensión de esto, haría falta más personal, si no hay más personal, lógicamente los centros se colapsan... [...]. Haría falta que todos los trabajadores sociales fuéramos a una, porque hay gente que... tiene muy claro lo que es la profesión y que da igual que te pongan con la aplicación informática que con las ayudas, sabe lo que tiene que hacer; $y$ hay gente que se pliega a este sistema, unas veces por... -a mi modo de ver- negligencia, y otras, por supervivencia, porque hay centros municipales que me consta que no es que sean negligentes, es que no pueden hacer más (E10).

En lo relativo a tiempos de espera, todos los profesionales manifestaban lo "clarísimamente excesivo" (E2) del tiempo de espera que los usuarios de servicios sociales municipales debían sufrir para ser atendidos. Esta situación, según varios entrevistados, es "una vergüenza" que “lamentablemente vamos normalizando" $\left(E_{5}\right)$.

La solución planteada por la Administración para descongestionar las listas de espera había sido una reducción en la duración de las citas con los usuarios, el ya nombrado 'plan de choque', lo que, según E1, estaba resultando insuficiente: "se agiliza temporalmente cuando nos exigen atender en veinte minutos, pero como la demanda sigue creciendo, dicha actuación ya está bloqueada". Sin embargo, estas medidas no han tenido los efectos deseados, pues al no reforzar el personal los centros y producirse numerosas bajas, las agendas de los profesionales se han visto bloqueadas:
Yo creo que más o menos... Ha habido como momentos de empujón, porque, por ejemplo, con el plan de choque ha habido unos momentos en los que, a lo mejor, ha podido ir un poquito más rápido en las primeras citas o en las primeras atenciones. Pero lo que pasa es que, si no se ha aumentado el número de personas que atiende, eso lo que ha llevado en algunos centros es a situaciones de bloqueo, o sea, a llevarnos al escenario opuesto, en el sentido de que ha habido compañeras que han tenido que cogerse bajas, bajas de larga duración, con lo cual las agendas se han distorsionado de una manera tremenda. Porque claro, lo que se hace es reubicar a esos usuarios en las agendas de otras compañeras, con la siguiente sobrecarga, y eso puede hacernos un efecto cascada (E11).

Por otra parte, cabe señalar la aportación que realiza el mismo entrevistado al señalar cómo la situación varía enormemente de un barrio a otro:

Es que ahí hay una variabilidad terrorífica en función del barrio. Que eso es algo que también se supone que se está trabajando para que no sea así, pero hay una diferencia abismal entre unos barrios que podemos encontrarnos con una espera..., yo creo que los últimos datos que estaban dando era de 22 días hábiles de tiempo de espera para una primera cita, hasta otros centros que puedes ser en una semana. 0 sea, 22 días hábiles quiere decir más de un mes. Entonces, claro, hay una diferencia bastante considerable... (E11).

Para mejorar esta situación, como ya apuntaba en su día E6, es necesario "invertir en recursos humanos, [porque] los que estamos ya no damos más de sî".

A los entrevistados se les pidió que evaluaran el funcionamiento del sistema de prestaciones y las respuestas de las administraciones ante la nueva situación; en este sentido, hay que diferenciar claramente las dos fases de la investigación. Inicialmente, todos coincidían en la denuncia de la mala gestión, concretamente del ingreso aragonés de inserción (IAI), que la administración pública estaba llevando a cabo: "en cuanto al IAI, la actuación de la Administración es absolutamente vergonzosa y obstructiva" (E2). Todos ponían el foco en lo inadmisible de los plazos de espera que debían sufrir los usuarios, "varios meses, cuando en teoría es un programa [pensado] como 'última opción', cuando ya se han agotado los de otros sistemas (empleo...)" (E3).

Por su parte, varios profesionales iban un poco más allá en la denuncia de este hecho, señalando en algunos casos cómo se estaba produciendo un auténtico incumplimiento de la ley, tanto por parte del Ayuntamiento de Zaragoza como del Gobierno de Aragón: "el IAl tiene un mes de concesión por ley y está tardando hasta doce meses. Por tanto, están cometiendo una irregularidad" (E5). 
Además del referido a los plazos en la espera, también existía un incumplimiento en cuanto a la falta de información a los ciudadanos sobre el recorte en las ayudas de urgencia: "la modificación de las $\mathrm{AU}$ [ayudas de urgente necesidad] fue por circular interna... Es que ahora son cuatro ayudas. ¿Dónde lo pone? Es que ha habido una circular interna..., pues a reclamar, porque en la ordenanza municipal no pone eso" (E8). Además preveían la inminente reforma del ingreso aragonés de inserción, lo que haría empeorar la situación, tal y como algunos vaticinaban: "lo que viene es una reforma del decreto del IAI, con lo cual mucha gente se queda fuera" $\left(\mathrm{E}_{7}\right)$.

En cuanto a la gestión pública de las ayudas de urgencia, existía mayor disparidad de opiniones. De este modo, mientras que $\mathrm{E}_{4}$ defendía que la Administración actuaba de manera similar que con el ingreso aragonés de inserción, E2 y E3 consideraban, respectivamente, que "aun siendo un período poco ágil, ha ido mejorando" y que "se han ido poniendo en marcha medidas que han disminuido el tiempo de resolución, que aún sería susceptible de mejorar".

Transcurridos más de dos años desde la investigación inicial, y con cambios en los Gobiernos autonómico y local, la situación respecto a la concesión de las prestaciones ha cambiado, como ya se ha puesto de manifiesto a lo largo de este análisis. En cuanto a las ayudas de urgencia, de competencia municipal, "el Ayuntamiento ha hecho un esfuerzo muy grande para acortar los tiempos de espera en las ayudas de urgencia, y las ayudas de alimentación están llegando casi a la semana, o sea, yo creo que está genial" (E4). Esta mejora no sólo es fruto del cambio de Gobierno local, ya que "empezó antes" (E10). Por el contrario, en lo relativo al ingreso aragonés de inserción, el cambio de Gobierno autonómico ha sido crucial para desatascar la situación de colapso que llevaban las solicitudes:

El tema de los IAls, pues sí que, coincidiendo con el cambio político (ahí sí que ha habido coincidencia), lo que os decía de retrasos de nueve meses a un año para una solicitud nueva, [ahora ha pasado] a dos o tres meses. Antes, [en] las renovaciones generalmente nadie se queda[ba] sin cobrar...; hubo un par de años que [estuvieron] tres cuatro meses sin cobrar" (E10).

En referencia a las respuestas de servicios sociales ante las nuevas necesidades y la idoneidad de éstas, todos los entrevistados coincidían en que era insuficiente: "si estamos diciendo que la demanda, a raíz de la crisis, aumenta, las respuestas tendrían que aumentarse, y es todo lo contrario lo que se está haciendo" (E6). Coincidían además en que las respuestas no se adaptaban a los cambios: "estamos actuando con medicamentos viejos para necesidades nuevas" $\left(E_{5}\right)$. Eran, por tanto, "estereotipadas y burocráticas, y no hay innovación ni intentos de adaptarse en ningún sentido" (E2).
Por su parte, E5 explicaba que los únicos intentos de adaptación provenían del esfuerzo individual de los profesionales: "lo nuevo [...] que se esté haciendo es porque cada profesional se busca la vida en atención más personal, más psicológica, motivación, etc. [...]. [Respecto a] las prestaciones sociales o las ayudas sociales para hacer frente a la crisis, no hay ninguna nueva, nada, nada". Por todo ello, algunos incidían en la importancia de realizar intervenciones más integrales, "planes entre áreas, por ejemplo, vivienda y servicios sociales, empleo y servicios sociales; coordinación exquisita entre Gobierno de Aragón y, en este caso, Ayuntamiento de Zaragoza” (E1).

En esta segunda etapa de la investigación, los profesionales coinciden en que las respuestas siguen sin ser adecuadas: "lo que atendemos es la urgencia, y sigue siendo muy asistencialista, por mucho que se pretenda hacer otra cosa" (E11). Por su parte, E12 afirma: "estamos haciendo un trabajo de bombero..., de apagar fuego". No se trataría tanto de un problema de falta de recursos económicos del sistema ${ }^{16}$, sino de un replanteamiento de las respuestas.

Para ello, en primer lugar, sería necesario más tiempo para detectar necesidades: "no tenemos el tiempo suficiente para poder hacer diagnósticos buenos, [y] se nos pueden escapar situaciones que están latentes. Un tema relacional de padres a hijos, que la crisis genera ansiedad, que la ansiedad la pueden pagar los hijos, todas esas cosas. [...] Hace falta tiempo de conexión personal” (E12). Aun así, se mantiene el esfuerzo por identificar esas situaciones, "sí que tratas de ver si hay [...] otro tipo de necesidades, otro tipo de situaciones, pero en estos momentos estamos tan desbordados que no se puede trabajar mucho más" (E11).

Ante la pregunta de la posible proliferación de iniciativas por parte de la comunidad -asociaciones de madres y padres de alumnos (AMPAS), asociaciones de vecinos, de inmigrantes-, en la primera investigación coincidían en que sí se estaba produciendo:

[Las] asociaciones de vecinos [están trabajando en] un tema muy interesante sobre vivienda infrautilizada (no ocupada) y la posibilidad de revertirla a la ciudadanía. Observo cómo las AMPAS se han unido y están ofreciendo materiales de otros años (libros, etc.) a otras familias [...]. Se están generando redes de apoyo mutuo entre personas que hacen de canguro, recogen a niños, etc. (E1).

No obstante, estas iniciativas presentaban tres características. Por una parte, la falta de capacidad económica, que las obligaba a "innovar con muy pocos medios” (E2). Por otra parte, según E4,

16 "Yo, para mí, en cuento a tiempo y en cuanto a dinero que se dedica, yo no tengo queja, yo creo que no podemos decir que se nos restringe nada" (E10). 
"no tienden a la autoorganización [...] y la participación de los afectados, sino que son de corte asistencialista (especialmente los proyectos de recogida y reparto de alimentos)". Por último, habrían surgido únicamente por la escasez de respuestas de la Administración, algo que no debería suceder:

Los bancos de libros, por ejemplo [...] es algo que ecológicamente [y] desde el punto de vista del consumo es mucho mejor, pero ha venido propiciado por la crisis y no por la conciencia ecológica [...]. Tendría que ser expresión de solidaridad, de vecindad..., pero no porque la Administración no lo haga (E5).

Además, se destacaban las iniciativas desarrolladas por los propios profesionales, como el Espacio de Información, Promoción y Defensa de los Derechos Sociales ${ }^{17}$.

Casi tres años más tarde, las iniciativas vecinales siguen desarrollándose: "el apoyo mutuo en la mayoría de los barrios de Zaragoza sí que funciona [...], sí que esa cultura de barrio sigue existiendo" (E11). No obstante, su presencia y actividad varía entre los distintos barrios de la ciudad. Así, mientras que E10 explica la existencia de un movimiento vecinal con una larga trayectoria, E12 señala únicamente ciertas iniciativas puntuales: "en los institutos sí que hicieron, y en algún colegio, como un mercadillo de libros de segunda mano, [...] cosas muy concretas en momentos muy determinados [...]. Pero más, no". Además, parece que en los últimos años las iniciativas ya existentes han continuado, pero no han aparecido otras nuevas: "las asociaciones de padres que guardaban [los libros] de un año para otro [...], pues, eso ahora, en vez de ser para dos familias solamente, ahora es para cincuenta familias. [...] Quiero decir que era un recurso que ya existía y [...] ha ido ampliándose, porque la demanda ha ido creciendo" (E11).

Sobre el papel de las entidades privadas en la acción social como posible complemento de los 'vacíos' del sistema, la mayor parte de los entrevistados afirmaban en la primera investigación que las entidades de acción social completaban la acción de los servicios sociales públicos, "pero no se les puede exigir que cumplan lo que la Administración, por ley, debería cubrir" (E6).

Sin embargo, algunos entrevistados expresaban que en ocasiones se generaban duplicidades, "como las que se dan en la actualidad [en alimentos, por ejemplo]", mientras que existía "carencia/ insuficiencia de otros [gastos sanitarios, formación, empleo]", lo que generaba "descontrol y que haya
${ }^{17}$ Se trata de un espacio comunitario formado por "personas preocupadas y afectadas por la vulneración reiterada en el acceso y la prestación de servicios sociales reconocidos como derechos sociales”. Para más información, véase 〈http://espacioderechos.blogspot. com>. que hacer además un extraordinario esfuerzo de coordinación por parte de todos" (E2).

En esta segunda etapa, las percepciones de los profesionales entrevistados coinciden en gran medida con las recogidas previamente. En primer lugar, señalan la importantísima labor de algunas entidades privadas, especialmente Cáritas, que "ha soportado en época de crisis, de fuerte crisis digo, porcentualmente, mucho más que la Administración [...]. A mí me parece que, sin ellos, no hubiera sido posible" (E12). Coincide en ello E11: "evidentemente Cáritas y El Refugio, fundamentalmente, son la otra gran pata de servicios sociales en nuestra ciudad en estos momentos".

No obstante y coincidiendo con las opiniones manifestadas en la primera fase, expresan que pueden generarse duplicidades en los servicios, con dos consecuencias. Por un lado, la posibilidad de que los usuarios 'acumulen' ayudas concedidas por distintas entidades, "no con ánimo de acopio, sino, pues bueno, 'nos organizamos, [y] cuantos más recursos haya, mejor”' (E10), lo que puntualmente puede dar lugar "a cosas muy curiosas, como trueques, como vendo esto, como...", tratándose, no obstante, de casos muy aislados, ya que "la mayor parte de la gente huye de eso". Por otro lado, esta duplicidad estaría generando situaciones de sobrecarga, no sólo de los profesionales, sino de los propios usuarios:

Si una persona está acudiendo a un servicio de empleo como el de Zaragoza Incluye, pues el hecho de que vaya a Cáritas a solicitar una ayuda de alimentación y también lo incluyan en su servicio de empleo [...], de alguna manera, a la persona también la estás sobrecargado en unos aspectos que a lo mejor no son necesarios (E11).

En cualquier caso, todos coinciden en que las acciones de entidades privadas nunca deberían sustituir a las realizadas por la Administración: "a mí lo que me molesta es si intuyo que la Administración quiere dejar de hacer cosas delegando en las entidades sociales" (E10).

En cuanto a la coordinación entre servicios sociales generales y entidades privadas de acción social, la mayoría de los entrevistados en la primera investigación afirmaban que sí se producía: "sí que hay una coordinación real, [...] es muy buena, de hecho, yo creo que lo que salva a los servicios sociales es la buena coordinación que hay" (E8). No obstante, E3 explicaba que se trataba de una coordinación "más informal que formal, y [que] no siempre [se producía]". Siguiendo esta línea, varios entrevistados exponían que dicha coordinación se producía únicamente por el esfuerzo y voluntad de los profesionales: "existen coordinaciones personales, profesionales, pero [...] basadas en la relación que tú estableces con ese compañero o compañera que trabaja en la zona" $\left(E_{5}\right)$. En este sentido, la coordinación distaba mucho del ideal, 
en opinión de varios entrevistados, incluyendo los pertenecientes a Cáritas. E9 afirmaba: "yo entiendo que coordinación es que todas las partes nos ponemos a trabajar en un plan común [...]. Pero eso no se puede poner en marcha, [...] y lo que hacemos es ir sumando ayudas". En aquel momento, la coordinación se encontraba, además, “muy centrada en el 'reparto' de la cobertura de las necesidades básicas” (E2).

Tres años más tarde, los profesionales manifiestan la existencia de distintos grados de coordinación con Cáritas, dependiendo del área de la ciudad:

Es mucho más complicado coordinarse [...] si estamos hablando de una zona en la que haya diez parroquias donde se atienda a una zona donde sólo haya dos. Porque entonces puedes hacer reuniones [...] una vez al mes, o cada dos meses, [...] porque puede haber derivaciones en ambos sentidos (E10).

También señalan que generalmente la coordinación es mejor en los barrios donde el Ayuntamiento desarrolla sus planes integrales, sobre todo en el ámbito de inserción laboral, educación de adultos o vivienda (Zaragoza Vivienda). Sin embargo, en los barrios donde no se implementan dichos planes, no existen canales de comunicación y coordinación tan estructurados, ni con entidades privadas, ni con otros sistemas, pudiendo existir "en algunos momentos reuniones macroárea, en los que sí puede haber cierta coordinación con algunos servicios, pero en cuanto a seguimiento de casos y todo esto, no" (E11).

En cualquier caso, la coordinación ha ido mejorando en los últimos años, como defiende E10: “sí..., hemos ido haciendo..., claro, hemos ido haciendo un camino, entonces sí que ahí vamos avanzando”. Ello ha mejorado la atención a los usuarios:

Hemos tenido muy buena experiencia cuando en estos casos de tanto tiempo de estar en el IAI..., 8 meses, de coordinarlos para sujetar a la familia, venga pues yo este mes intento pagar el alquiler por ejemplo, y vosotros el mes que viene... no duplicar ayudas para posibilitar que la familia más, mejor atendida... (E11).

Por último, al preguntar si se estaban desatendiendo otras necesidades, casi todos respondían afirmativa y rotundamente. E5 explicaba que se estaba "intentando contener la situación con las ayudas de urgencia, con las ayudas para alquileres y las ayudas para alimentación, principalmente”. De este modo, "la presión del día a día y de la agenda [hace que] no nos detengamos lo suficiente para crear un buen espacio para la relación de ayuda” (E1), situación que afectaba a los usuarios "para el tema de motivación, para el tema emocional, para el tema de la formación, del reciclaje..." (E5).

Además, las circunstancias repercutían en el desarrollo profesional de los trabajadores sociales:
Se pierde la visión integral de la persona. Una persona es mucho más que los ingresos que tiene. Es sus emociones, su sentido vital, su proyecto, el vacío al que se asoma cada mañana cuando no ve un futuro, el control sobre su vida. En ese sentido, creo que atender a estos aspectos es, en muchos momentos, tan importante como garantizar el pago del alquiler (E4).

Según la información recogida para esta segunda investigación, no parece que la situación haya cambiado. Como explica E12, las ayudas económicas siguen siendo muy necesarias, pero resultan insuficientes: "cuando una persona está teniendo necesidad de comida, no puedes plantearte otras historias [...]. Pero a la vez, puedes [...] generar otro tipo de respuestas a otras necesidades que pueda haber".

Cabe destacar que en estos años, a pesar de las dificultades, se han ido detectando nuevas necesidades, derivadas fundamentalmente de los desempleos de larga duración, haciéndose visibles situaciones que se encontraban, de alguna manera, ocultas en familias aparentemente normalizadas:

Mucha gente tenía otros problemas, [pero] más o menos se sujetaban, porque había trabajo, y el trabajo y el dinero tapa mucho. Cuando eso falla, se desmoronan relaciones familiares, surgen temas que a lo mejor no habían surgido, [por ejemplo] el tema del consumo de tóxicos (E10).

Muchas de estas necesidades derivan del desgaste (emocional, anímico, relacional) provocado por la situación prolongada de desempleo:

Ha habido gente que se ha pegado cinco años en paro, hasta que ha enganchado con algo..., pues eso es pasar por todas las fases. También hay una fase de resignación, y también ahí hay una fase de acompañar eso [...], favorecer que la otra persona no pierda la esperanza y que siga activo y siga esperando que pueda cambiar algo. Es que es lo que hay, porque ni está en su mano, ni está en la mía (E10).

A todo ello se añade que también se han ido dejando de lado otros aspectos “más sociales y más de dinamización comunitaria” (E11).

Los entrevistados achacan estas carencias a la falta de tiempo, que se traduce en dos situaciones. Por un lado, el aplazamiento de la atención de necesidades menos urgentes:

Hay unas prioridades imperiosas [...], por lo cual, estas otras cosas que te gustaría hacer o a las que te gustaría llegar, generalmente, se quedan [...] para cuando podamos, y ese 'cuando podamos' generalmente es difícil que llegue en estas circunstancias (E11).

Por otro, el aplazamiento de sus propias necesidades formativas como profesionales, para adquirir nuevas 
herramientas, al estar las actuales "desfasadas, desfasadas completamente”, como señala E12, que añade:

De repente..., orienta..., o logra itinerarios de inserción a [...] esa mujer que ha acabado Trabajo Social..., que la diferencia es que ella no tiene trabajo, y yo sí. ¿Cómo lo hago? Tampoco han dejado tiempo de formación..., porque... también la tenemos limitada en el trabajo, porque claro, si tienes que estar atendiendo...

\subsection{Valoración global y propuestas}

En este apartado, se presenta la valoración global de los entrevistados sobre la situación de servicios sociales.

Aunque no había consenso en la primera fase, cabe señalar que varios entrevistados señalaban la insuficiencia de recursos como uno de los principales problemas de los servicios sociales. E4 hablaba de "colapso" y "falta de recursos"; E3 especificaba que hay "escasez secular de recursos humanos y económicos"; y E1 hacía referencia a que "no existe dotación personal suficiente [...] para hacer frente con garantías y con corrección a la situación actual”.

Otro punto destacable son las dificultades organizativas o de planificación. E4 afirmaba que "falta [...] orientación y sentido estratégico", porque "no nos adelantamos a los acontecimientos, no se planifica, investiga, indaga”. Opinión muy similar mantenía $E_{5}$, denunciando que "tendría que haber una planificación para hacer frente a la crisis, que no la hay".

Un tercer problema mencionado en las entrevistas se refiere a la intervención, en la que, según E3, primaba una "atención a demanda sobre atención integral”, porque "existe una "concepción únicamente prestacionista", debido a la "falta de trabajo por proyectos” y “de orientación comunitaria” (E4).

En esta segunda fase, los entrevistados señalan como uno de los problemas fundamentales la tensión creciente en algunos centros de la ciudad: “creo que los servicios sociales tienen que ser un lugar de acogida, y muchas veces veo que son un lugar de conflicto, de discusión, de malos entendidos” (E10). Esta situación puede deberse a varios motivos. En primer lugar, a las propias “crisis profesionales" (E10), derivadas de la presión generada por la sobrecarga de trabajo, la falta de herramientas adaptadas a las nuevas necesidades o la carencia de recursos humanos suficientes y su mal aprovechamiento, como afirma E11, "no solamente faltan, sino que no se están aprovechando al cien por cien”, al no existir puesta en común de los conocimientos adquiridos. En segundo lugar, los usuarios acuden a los centros cada vez con mayor conocimiento y exigencia de sus derechos, situación que ha ido modificando la relación entre el usuario y el profesional:
La gente viene muy exigente, y a mí me parece bien, lo entiendo [...]. Está cambiando, la gente no es la actitud de... 'yo no sé mis derechos', [ahora es] 'yo tengo mis derechos, quiero esto, esto lo entiendo, esto y por qué no...' (E10).

Estas nuevas dinámicas también generan conflictos en el rol del propio trabajador social, probablemente causadas, en parte, al desgaste existente: "hay mucho[s] profesional[es] [a los] que les sabe mal que se les reclamen ayudas o que tenga[n] que dar explicaciones" (E10). Esta tensión ha llegado a desembocar incluso en agresiones puntuales, como explica E12: "si una persona me viene muy nerviosa porque no tiene para comer, porque lleva tanto tiempo esperando el IAI [...] y yo digo 'ése no es mi problema' [...]. Yo puedo generar que me den una leche, [aunque] yo no justifico que me la den”.

Con todo ello, se están creando "centros enfermos [en los que] no se funciona bien en equipo [...], [que] no consiste sólo en reunirse", centros donde "el ambiente podría mejorarse” (E10). Como señalaba un entrevistado, "la higiene mental es fundamental, [y] es verdad que hay centros [en los] que esto no ocurre [...]. Depende un poco del ambiente, de las personas" (E12). También la salud de los propios profesionales se está resintiendo: "tienes que tener una fortaleza mental, [...] para que yo atienda bien, tengo que estar bien, y el índice de personas con baja en el servicio ha aumentado" (E12).

Por otra parte, los entrevistados hacen de nuevo hincapié en que las respuestas no se encuentran adaptadas las nuevas necesidades. Ante esta situación,

sí que hay una buena disposición hacia el cambio por parte del equipo de Gobierno, pero hay muy pocas posibilidades reales de efectuar estos cambios [...]. Uno de nuestros principales problemas es la burocratización tan extrema que tienen los servicios sociales dentro del Ayuntamiento de Zaragoza (E11).

Por último, se ha señalado una visión demasiado cuantitativa de la tarea de los trabajadores sociales, criticándose "que se cuantifique tanto el número de citas, el número de ayudas, y que no te permitan hacer una intervención un poquito más amplia" (E11).

En cuanto a las propuestas de mejora en los servicios sociales, en la primera investigación existía disparidad de respuestas. Así, E4 proponía de manera general "aprovechar el gran potencial de los profesionales y la experiencia acumulada y poner[se] a pensar”. Otros eran más concretos, como E2, que proponía “una organización basada en programas, con objetivos de crecimiento personal y puesta en valor de lo que pueden [sic.] ofrecer la ciudadanía, y no en función de lo que se les [sic.] pueda dar". E1 sugería "hacer sesiones grupales, ya que hay muchos colectivos que cumplen el mismo perfil (por ejemplo, los perceptores del IAI)”. En esa línea, E7 
proponía "hacer trabajo comunitario, [ya que] es muy preventivo, [y] con la prevención evitamos el volumen de atención". Finalmente, E2 planteaba "crear mecanismos que favorezcan la coordinación; [...] no duplicar entidades, programas, actuaciones... entre entidades públicas y privadas, sino procurar una planificación a nivel global de los servicios sociales".

Por otra parte, algunos entrevistados daban importancia a la atención a la vivienda: "hay que [...] conseguir que los colectivos con mayores dificultades puedan acceder a vivienda protegida" (E1); "[se ha de] potenciar la vinculación del sistema de servicios sociales con los sistemas de empleo y vivienda" (E2). Otro entrevistado proponía incluso "sacar todo el dinero de servicios sociales [...], porque además condiciona mucho la relación [entre profesional y usuario]" (E8). En definitiva, se trataba de "dejar de ser reactivos ante la situación, y ser proactivos" (E4).

En esta segunda etapa, los entrevistados expresan que se han realizado algunos avances en la línea de las propuestas de la etapa anterior. E10 explica: "para mí una novedad ha sido el Zaragoza Incluye, que es la primera vez que en servicios sociales hay un proyecto de empleo". No obstante, más allá de este programa, afirma que los servicios sociales "no está ofreciendo cosas diferentes".

En este sentido, y a partir de la formación del nuevo Gobierno, E11 señala que "se está trabajando en los programas específicos, [...] los protocolos de funcionamiento de menores, de prestaciones domiciliarias, y [...] el de información, orientación y valoración", siendo una de sus propuestas "que se clarifique realmente cuál es el modelo de funcionar".

En esta nueva ronda de entrevistas, una de las propuestas lanzadas por los profesionales se centra en plantear respuestas más adaptadas a las nuevas necesidades,

más activas, [...] donde [el usuario] reciba dinero y [...] esté activo, ya sea a través de un contrato de empleo o de otro tipo de acción [...], y no en el sentido de que te lo tienes que sudar para llevarte el dinero [...], sino en el de que tú recobres tu dignidad, que no la has perdido, pero que la sientas (E11).

Para plantearlas, sería conveniente, según E12, que existiese más formación para los profesionales, "reciclaje también [para] saber dar respuestas con herramientas adecuadas y actualizadas”. Además, sería positiva la creación de "espacios para que todos los trabajadores pudieran aportar sus propuestas, porque creo que hay gente muy válida [...], que conoce muy bien sus zonas de referencia, y que [...] pudiera hacer propuesta[s] de cara a la dinamización de áreas concretas” (E11).

Por otra parte, proponen iniciativas que podrían repercutir en la mejora del ambiente de los centros, ya que, como defiende E10:
[El] centro de servicios sociales tiene que ser un lugar amable, donde te sientes bien, donde puedes confiar, y donde, aparte del dinero, te llevas esperanza, te llevas confianza. Entonces, eso ¿cómo se favorece? Se favorece con menos presión a los profesionales [...], con más apoyo en general.

Una de las propuestas para reducir esta presión pasaría por aumentar el personal de los centros: "hace falta más personal [...]. Si sólo quieres una gestión pura y dura, esto se organiza y podemos sacar estas ayudas, sin más. Si quieres algo más, hace falta más personal” (E10). En esta línea, E1o plantea la posible implementación de un sistema de cribaje previo, bien realizado por trabajadores sociales o por técnicos, una idea que parece que está barajando la propia Administración: "en cuanto a lo que era la información y orientación inicial, se planteaba [que] tenía que haber como una trabajadora social de primera acogida" (E10). Coincide con ello E12: "si yo no hago tanto [trabajo] burocrático, tanto [trabajo] administrativo... [...]. No es tanto que se necesiten más trabajadores sociales, pero tenemos [que ver] cómo se puede organizar mejor".

Con estas medidas, los entrevistados creen que podrían desarrollar mejor sus capacidades profesionales. E10 argumenta que "hay muchos profesionales haciendo un esfuerzo por ofrecer algo más". Como ejemplo, se habla de la posibilidad de realizar más trabajo en grupo y comunitario: “yo, por ejemplo, [...] sería feliz con hacer un grupo [con] la cantidad de mujeres que veo con el síndrome del cuidador por la atención a personas dependientes", 0 "poner en contacto [a] personas que necesitan trabajar en casa de personas mayores con gente que sabemos que [las] necesitan", o también "organizar sesiones de formación" (E12). Con estas iniciativas, se generaría en los trabajadores sociales "más vidilla y más satisfacción personal” (E12). Ahora mismo, según E11, "esto no es trabajo social, esto es escuchar a las personas, que me firmen una hoja que yo amontono. ¿En qué posición me sitúa a mí como trabajadora social?".

En definitiva, se propone que se "cuide evidentemente a los usuarios, pero [que también] se cuide a los profesionales de servicios sociales" (E12), idea en la que la Administración ya se encuentra trabajando:

Ahora mismo se están haciendo estudios psicosociales [...] en los distintos puestos de trabajo [...]. Ha habido solicitudes directas desde los propios centros, y también desde el propio servicio de prevención y salud laboral [...], porque realmente ha habido muchas bajas por estos temas de sobrecarga y ansiedad dentro de servicios sociales. [...] Lo que se puede aportar [en] un equipo sano va a ser muchísimo más que en un equipo distorsionado (E11).

Por último, se propone una atención más homogénea entre barrios: "que de alguna manera fuese más 
equilibrada para los usuarios, fuesen del barrio que fuesen, porque en este momento sí que hay diferencias" (E11).

\section{Conclusiones}

\subsection{Usuarios}

En el periodo analizado, se asiste a un aumento de los usuarios, que se traduce en mayor carga de trabajo para los profesionales. Se distinguen dos colectivos: 'tradicionales' y 'nuevos'. Estos últimos, o bien habían acudido en alguna ocasión a los servicios sociales municipales, 0 , por el contrario, eran nuevos completamente en el sistema. Cabe señalar las diferencias existentes entre ambos grupos: los tradicionales se caracterizan por presentar, además de problemas económicos, otro tipo de problemas personales o sociales que les dificultan llevar una vida autónoma, organizada y propia. El denominador común de los nuevos usuarios es la falta de ingresos como consecuencia de una situación de desempleo de larga duración y un agotamiento de la protección social. Unido a este problema inicial, en la segunda fase de la investigación se observa que, además, presentan problemas emocionales o psicológicos, que a largo plazo pueden derivar en otros problemas, como apuntan algunos profesionales. La diferenciación entre ambos grupos de usuarios persiste, aunque bien es cierto que la situación de los usuarios 'tradicionales' de servicios sociales se ha visto cronificada.

La falta de empleo y, por ende, la ausencia de ingresos es el principal motivo de llegada de los nuevos usuarios a los servicios sociales municipales, aunque ahora, como ya se ha señalado, el deterioro emocional ya supone un problema destacable. Además, este nuevo colectivo tiene mayor información sobre los servicios sociales o sobre lo que allí se ofrece.

En todos los usuarios prevalecen las necesidades básicas frente a las de otro tipo, lo que da lugar a un abandono de la atención integral, así como a un aumento de las prestaciones económicas, en detrimento de otro tipo de intervenciones. Se ha disparado el número de ayudas de urgencia, y hoy en día, además de cubrir déficits habituales (luz, agua, alquiler), crecen los problemas derivados de impago de hipotecas.

Además de las dificultades que atraviesa el propio sistema de servicios sociales, se observa cómo problemas derivados de otras áreas repercuten sobre los servicios sociales, siendo éstos incapaces de darles respuesta.

\subsection{Capacidad de respuesta de los servicios sociales a las demandas actuales}

En general, se dedica poco tiempo a la atención de los usuarios, que está estipulada en veinte minutos a partir de una directriz, aunque bien es cierto que no todos los profesionales la cumplen. Éste no es el único problema detectado, puesto que la falta de recursos, unida a los déficits de la organización del propio sistema, dificultan el trabajo diario de los profesionales. Otro aspecto negativo que cabe destacar es el excesivo tiempo de espera en la atención, aunque existen diferencias notables entre un centro y otro.

Respecto a la actuación de la Administración en la concesión de diferentes prestaciones (fundamentalmente, ayudas de urgente necesidad e ingreso aragonés de inserción), es importante señalar las diferencias observadas en el periodo analizado. En la primera fase de la investigación, se ponía el foco básicamente en los excesivos retrasos por parte del Gobierno autonómico (en la concesión del ingreso aragonés de inserción). En la segunda fase, la situación ha mejorado debido, en parte, al cambio de Gobierno producido en mayo de 2015.

A pesar de estos tímidos avances, es preciso un replanteamiento general de los servicios sociales, puesto que no se adaptan a las nuevas necesidades de los usuarios y la intervención que realizan es principalmente de tipo paliativo.

El surgimiento de iniciativas comunitarias ha ayudado a amortiguar las dificultades detectadas, aunque en la actualidad esa situación se ha estancado, es decir, se mantienen ciertas iniciativas, pero no surgen nuevas. También ha sido determinante el papel de las entidades privadas de acción social, que en algunos casos ha ayudado a cubrir vacíos de las administraciones públicas, pero en otros ha provocado duplicidades en la atención.

\subsection{Valoración global y propuestas}

Por último, en cuanto a los problemas detectados, inicialmente los profesionales señalaban la insuficiencia de recursos, la falta de organización y de planificación, así como el tipo de intervención desarrollada (atención a demanda frente a atención integral). Son interesantes los problemas detectados en la segunda fase. Se puede afirmar que en algunos centros la tensión crece, debido a las propias crisis profesionales (presión, falta de herramientas o insuficiencia de recursos), además de a un conocimiento cada vez mayor por parte de los usuarios, modificándose en este sentido la relación entre el trabajador social y el usuario. También hay que remarcar que la salud de los profesionales está viéndose afectada, y así nos lo ponen de manifiesto.

Por todo ello, las propuestas que se desprenden del análisis realizado van en sintonía con los problemas detectados. En la primera fase, los trabajadores sociales consideraban que cambios en la organización, la puesta en marcha de sesiones grupales y la mejora de la coordinación, entre otros, ayudaría a corregir los déficits del sistema. 
En la segunda fase, se aprecia cómo algunos cambios ya han tenido lugar, sobre todo a partir de la formación del nuevo Gobierno local ${ }^{18}$. Sin embargo, todavía hay camino que recorrer en cuanto a la adaptación de recursos a las nuevas necesidades, así como a la mayor formación para los profesionales, sin olvidarnos, evidentemente, de mejoras respecto a recursos. Un aumento de éstos podría suavizar la tensión de los centros, a la vez que la de los propios profesionales. 
ABAD MIGUÉLEZ, B.; y MARTÍN ARANAGA, I. (2015): “El Trabajo Social ante la crisis. Nuevos retos para el ejercicio profesional de los y las trabajadoras sociales", Cuadernos de Trabajo Social, vol. 28, nํㅜ 2, págs. 175-185.

ASOCIACIÓN ESTATAL DE DIRECTORAS Y GERENTES DE SERVICIOS SOCIALES (2016): Índice DEC de desarrollo de los servicios sociales, Asociación Estatal de Directoras y Gerentes de Servicios Sociales.

AGUILAR HENDRIKSON, M. (2013): "Los servicios sociales en la tormenta”, Documentación Social, nำ166, págs. 145-167.

ALGUACIL GÓMEZ, J. (2012): “La quiebra del incompleto sistema de Servicios Sociales en España", Cuadernos de Trabajo Social, vol. 25, $\mathrm{n}-1$, págs. 63-74.

ARAGÓN (2009): “Ley 5/2009, de 30 de junio, de Servicios Sociales de Aragón", Boletín Oficial de Aragón, no 132 , 10-7-2009, págs. 18.244-18.280 [<http://www.boa.aragon.es/cgi-bin/EBOA/BR SCGI?CMD=VEROBJ\&MLKOB=390353230505'].

AYALA, L.; y RUIZ-HUERTA, J. (2015): "Estado de bienestar y políticas sociales:tendencias y perspectivas", en BLANCO, A., Informe España 2015, Madrid, Fundación Encuentro, págs. 250-279.

BARBIERI, N.; y GALLEGO, R. (2015): "El despliegue de la Ley de Dependencia en el País Vasco y la Comunidad de Madrid (2007-2012)", Zerbitzuan, no 6o, págs. 93-111 [khttps://doi. org/10.5569/1134-7147.60.07)].

BARRIGA, L. (2014): “Las políticas de austeridad y el horizonte de la reforma de la Administración Local: un cerco a los servicios sociales en España”, Documentación Social, nํㅜ 175, págs. $65-103$
DEUSDAD, B. (2016): “Restructuring long-term care in Spain: The impact of the economic crisis on social policy and social work practice", Journal of Social Service Research, $\mathrm{n}^{0}$ 42, págs. 246-262.

D. L. G. (2016): “Zaragoza concede ayudas a más de mil niños para comedor y libros", El Periódico, 16-11-2016 [<http://www.elperiodicodearagon. com/noticias/aragon/zaragoza-concedeayudas-mas-mil-ninos-comedorlibros_1159660.htm〉].

DUQUE CARRO, J. M. (2012): “Redescubrimiento de los servicios sociales de atención primaria: hacia un (nuevo) modelo de atención personal y comunitaria", Zerbitzuan, no 52 , págs. 23-44 ['https://doi.org/10.5569/1134-7147.52.02'].

FANTOVA, F. (2016): "Nuevos enfoques para los servicios sociales ante la nueva realidad social", Revista Española del Tercer Sector, nํ 33, págs. 113-139.

FUNDACIÓN FOESSA (2013): Desigualdad y derechos sociales, Madrid, Fundación Foessa.

- (2012): Exclusión y desarrollo social, serie Análisis y Perspectivas, Madrid, Fundación Foessa.

FUNDACIÓN 1ํDE MAYO (2012): Trabajadores pobres y empobrecimiento en España, colección Estudios, $\mathrm{n} \div 56$.

LAPARRA, M.; y PÉREZ ERANSUS, B. (2012): Crisis y fractura social en Europa. Causas y efectos en España, Barcelona, Obra Social La Caixa.

LIMA, A. (2013): I Informe sobre los servicios sociales en España, Madrid, Consejo General del Trabajo Social.

LÓPEZ CASASNOVAS, G. (2015): El bienestar desigual, Barcelona, Península. 
MAREA NARANJA ARAGÓN. (2012): Situación social en Aragón 2012. Personas, familias y sociedad, Marea Naranja Aragón.

MARTÍNEZ VIRTO, L.; y PÉREZ ERANSUS, B. (2015): “La austeridad intesifica la exclusión social e incrementa la desigualdad (aproximación a las consecuencias de los recortes en servicios sociales a partir de la experiencia en Navarra)", Revista Española del Tercer Sector, n- 31, págs. 65-88.

MONTSERRAT, J. (2015): “Impactos de las medidas de estabilidad presupuestaria en el Sistema de la Autonomía y Atención a la Dependencia: retos de futuro", Zerbitzuan, no 60, págs. 9-30 ['https://doi.org/10.5569/1134-7147.60.02〉].
OBSERVATORIO ESTATAL DE LA DEPENDENCIA (2012): Impacto económico de la reforma de la Ley de la Dependencia, Asociación Estatal de Directoras y Gerentes de Servicios Sociales.

RODRÍGUEZ CABRERO, G. (2012): Servicios sociales y cohesión social, Madrid, Consejo Económico y Social.

RODRÍGUEZ RODRÍGUEZ, P. (2015): La situación del Servicio de Ayuda a Domicilio en el ámbito local y perspectivas de futuro, Madrid, Federación Española de Municipios y Provincias.

SANZO, L. (2013): "La crisis de principios del siglo XXI en España”, Documentación Social, nํㅜ166, págs. 15-43. 